\title{
Exclusive tongue tip reconstruction of hemiglossectomy defects using the underrated lateral arm free flap with bilobed design
}

\author{
Jeongseok $\mathrm{Oh}^{1 *}$, \\ Tae Hyeon Lee ${ }^{1 *}$, \\ Jang Hyun Lee ${ }^{2}$, \\ Kyung Tae ${ }^{3}$, \\ Seong Oh Park ${ }^{1}$, \\ Hee Chang Ahn ${ }^{1}$
}

${ }^{1}$ Department of Plastic and Reconstructive Surgery, Hanyang University Seoul Hospital, Hanyang University College of Medicine, Seoul; ${ }^{2}$ Department of Plastic and Reconstructive Surgery, Hanyang University Guri Hospital, Hanyang University College of Medicine, Guri; ${ }^{3}$ Department of Otolaryngology-Head and Neck Surgery, Hanyang University College of Medicine, Seoul, Korea

\begin{abstract}
Background: Tongue reconstruction is challenging with the unique function and anatomy. Goals for reconstruction differ depending on the extent of reconstruction. Thin and pliable flaps are useful for tongue tip reconstruction, for appearance and mobility. This study reports lateral arm free flap (LAFF) as a safe and optimal option for hemi-tongue reconstruction, especially for tongue tip after hemiglossectomy.

Methods: Thirteen LAFFs were performed for hemi-tongue reconstruction after hemiglossectomy from 1995 to 2018. Of the 13 patients, seven were male and six were female, age varying from 24 to 64 years.

Results: All flaps healed uneventfully without complications. Donor sites were closed primarily. The recipient vessels for microvascular anastomosis were mainly superior thyroidal artery, external jugular vein. All patients returned to normal diet, with no complaints regarding reconstructed tongue and donor site.

Conclusion: The LAFF is hairless, thin (especially with lateral epicondyle approach), and potentially sensate. They are advantageous features for tongue tip and hemi-tongue reconstruction. Donor site sacrifices the inessential posterior radial collateral artery, and the scar is hidden under short sleeve shirts. We believe that LAFF can be considered as the first choice flap for hemitongue reconstruction, over radial forearm free flaps.
\end{abstract}

Keywords: Neoplasms / Reconstructive surgical procedures / Surgical flaps

\section{INTRODUCTION}

Tongue cancer is the most common oral cancer with reports of incidence of 3.0 per 100,000 individuals [1]. Multidisciplinary treatment with radical resection and radiation therapy is recommended $[2,3]$. Tongue reconstruction involves reproducing the complex anatomy, function, bulk, texture, mobility, and

\section{Correspondence: Hee Chang Ahn}

Department of Plastic and Reconstructive Surgery, Hanyang University Seoul

Hospital, 222-1 Wangsimni-ro, Seongdong-gu, Seoul 04763, Korea

E-mail: ahnhc@hanyang.ac.kr

*The first two authors contributed equally to this work.

Received July 5, 2018 / Revised October 31, 2018 / Accepted November 2, 2018 sensation [4]. With microsurgical advancement, many options are available for tongue reconstruction [5]. The challenge nowadays is more about optimization than success.

Current concepts of tongue reconstruction classify tongue defects as hemiglossectomy, subtotal, and total [6,7]. Flap choice is suggested to be made based on bulk and motility according to the defect [8]. For hemiglossectomy defects, the radial forearm free flap (RAFF) is recommended for its thin pliable nature with the long constant pedicle. Thin anterolateral thigh free flaps (ALTFFs) are also recommended as an alternative option. Preservation of tongue mobility with a small amount of bulk is considered the key to success of such defects [6-8]. 
Lateral arm free flap (LAFF), first introduced in 1982 by Song et al. [9], is well known for the thin pliable tissue. It remains underused with reports of inconsistent perforator anatomy, short pedicle, small vessel caliber, and difficult flap dissection [10]. But, LAFF is thin (advantageous for tongue motility), hairless, potentially sensate [11]. Unlike what is known, LAFFs can be harvested reliably based on relatively consistent perforators [12]. Donor site can be closed easily with the amount of tissue needed for reconstruction of hemiglossectomy defects. In this study, based on our results, we report LAFF as the safe and exclusively optimal choice for hemiglossectomy defect reconstruction.

\section{METHODS}

A retrospective review of 13 patients that underwent hemiglossectomy and hemi-tongue reconstruction from 1995 to 2018 was performed. Demographic and operative data were collected (Table 1). LAFF was performed by a single plastic surgeon (HCA) in all cases. The bilobed design was used for tongue tip and mouth floor reconstruction. Reconstructed tongue and donor site was evaluated. The follow-up period ranged from 2 months to 90 months. Neurorrhaphy using a posterior antebrachial cutaneous nerve was performed in four cases.

\section{Surgical technique}

A patient is placed in a supine position. The nondominant arm was selected. A line is drawn between the lateral epicondyle and deltoid muscle insertion. The lateral intermuscular septum, located $1 \mathrm{~cm}$ posterior to this line, becomes the central axis of the flap. The flap is designed including the axis as circular, bilobed, or elliptical, depending on the defect. In the bilobed design flap, each lobe is used to reconstruct tongue tip defects and mouth floor defects. Design a lobe for the tongue tip defect near the lateral epicondyle to obtain the thinner flap. Incision is made on the anterior margin of the flap. Suprafascial dissection is made until the lateral intermuscular septum, posterior collateral radial artery and its perforators are identified. Muscular perforator branches are ligated. Radial nerve should be preserved, so the posterior antebrachial cutaneous nerve can be used to for sensate flap harvest. Posterior incision of flap is made, flap is elevated. Proximal dissection provides longer pedicle and larger caliber. De-epithelization and defattening is performed according to defect size and flap inset. Donor site is closed primarily. The recipient vessels for microvascular anastomosis were mainly superior thyroidal artery, external jugular vein.

\section{RESULTS}

Demographics, defect type, and complications are summarized in Table 1. All flaps healed uneventfully without complications. No patients experienced infection, hematoma, fistulas or donor-site complications. Donor sites were closed primarily. LAFF was used for hemi-tongue and mouth floor. All patients returned to normal diet, with no complaints regarding reconstructed tongue and donor site. All flaps survived without further intervention. All patients were satisfied with the functional and aesthetic outcome.

\section{Case 1}

The first case is a 37-year-old male patient who diagnosed recurred tongue cancer (Fig. 1A). A LAFF was performed after left hemiglossectomy by ENT (ear, nose, and throat) department (Fig. 1B). A right-handed patient, the bilobed LAFF of about $12 \times 9 \mathrm{~cm}$ was designed to the left lateral arm. Upper lobe is for the tongue tip and hemiglossectomy defect and lower lobe for the mouth floor defect (Fig. 1C). The posterior radial collateral

Table 1. Demographics, defect type, and surgical procedures of hemi-tongue reconstruction patients

\begin{tabular}{|c|c|c|c|c|c|c|c|c|}
\hline Sex & Age (yr) & Diagnosis & Neurorrhaphy & Recipient vessel & Design & Operation & Complication & Donor site \\
\hline $\mathrm{F}$ & 38 & Hemiglossectomy defect & $\times$ & Sup thyroidal a Ext jugular v & Bilobed & LAFF & None & Primary closure \\
\hline M & 34 & Hemiglossectomy defect & $\bigcirc$ & Sup thyroidal a Ext jugular v & Bilobed & LAFF & None & Primary closure \\
\hline $\mathrm{F}$ & 35 & Hemiglossectomy defect & $x$ & Sup thyroidal a Ext jugular v & Bilobed & LAFF & None & Primary closure \\
\hline M & 37 & Hemiglossectomy defect & $\bigcirc$ & Lingual a lingual v & Bilobed & LAFF & None & Primary closure \\
\hline M & 35 & Hemiglossectomy defect & $\times$ & Sup thyroidal a Ext jugular v & Bilobed & LAFF & None & Primary closure \\
\hline $\mathrm{F}$ & 41 & Hemiglossectomy defect & $\times$ & Sup thyroidal a Ext jugular v & Bilobed & LAFF & None & Primary closure \\
\hline M & 64 & Hemiglossectomy defect & $\times$ & Sup thyroidal a Ext jugular v & Bilobed & LAFF & None & Primary closure \\
\hline$F$ & 34 & Hemiglossectomy defect & $\times$ & Sup thyroidal a Ext jugular v & Bilobed & LAFF & None & Primary closure \\
\hline M & 44 & Hemiglossectomy defect & $\times$ & Sup thyroidal a Ext jugular v & Bilobed & LAFF & None & Primary closure \\
\hline $\mathrm{F}$ & 24 & Hemiglossectomy defect & $\times$ & Sup thyroidal a Ext jugular v & Bilobed & LAFF & None & Primary closure \\
\hline $\mathrm{F}$ & 62 & Hemiglossectomy defect & $\bigcirc$ & Sup thyroidal a Ext jugular v & Bilobed & LAFF & None & Primary closure \\
\hline M & 52 & Hemiglossectomy defect & $x$ & Sup thyroidal a Ext jugular v & Bilobed & LAFF & None & Primary closure \\
\hline M & 62 & Hemiglossectomy defect & $\bigcirc$ & Sup thyroidal a Ext jugular v & Bilobed & LAFF & None & Primary closure \\
\hline
\end{tabular}

F, female; M, male; Sup, superior; a, artery; Ext, external; v, vein; LAFF, lateral arm free flap. 

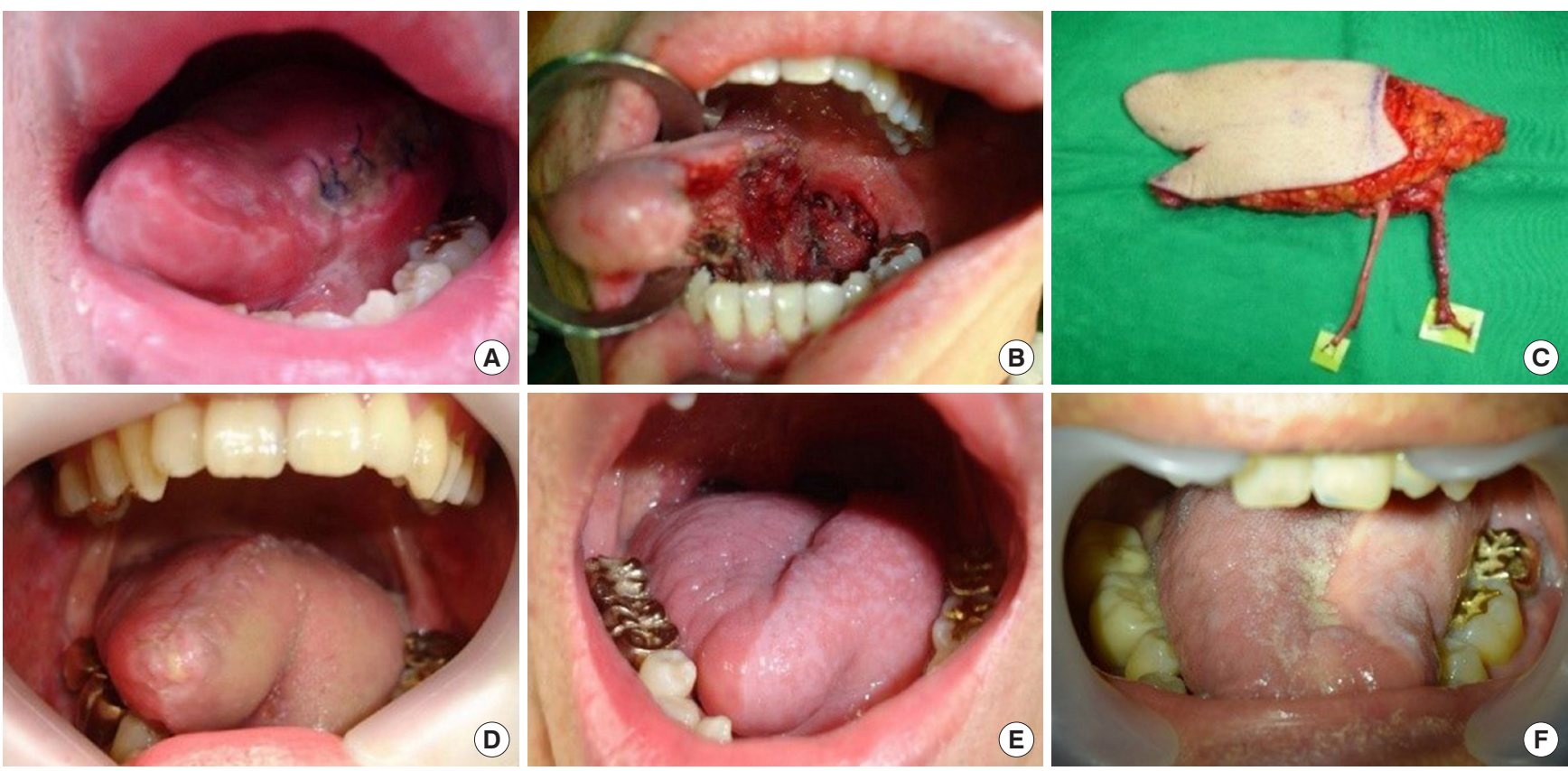

Fig. 1. Case of left hemi-tongue reconstruction using bilobed lateral arm free flap. (A) Preoperative photograph shows biopsy confirmed left tongue cancer. (B) Intraoperative photograph after left hemiglossectomy was performed by the ENT (ear, nose, and throat) department. (C) Intraoperative photograph of harvested bilobed lateral arm free flap. Upper lobe is for the tongue tip and lower lobe for the tongue base. Small yellow background identifies posterior antebrachial cutaneous nerve. Large yellow background identifies posterior radial collateral artery and its vena comitans, which is used as the main pedicle. (D) One-month postoperative photograph. (E) Eleven-month postoperative photograph. (F) Eight-year postoperative photograph shows mucosalization of the reconstructed tongue.

artery and vena comitantes were anastomosed lingual artery and vein, respectively. The posterior antebrachial cutaneous nerve was anastomosed to the lingual nerve. Postoperatively, the flap was well taken without complications on both the donor site and the recipient site. At 1 month postoperatively (Fig. 1D), the patient complained of tasting, swallowing, and pronouncing difficulty, but subjective symptoms improved at 11 months postoperatively (Fig. 1E). Postoperative follow-up after 8 years revealed no recurrence and showed mucosalization of the reconstructed tongue (Fig. $1 \mathrm{~F})$.

\section{Case 2}

The second case is a 24-year-old female patient diagnosed with tongue cancer (Fig. 2A). Right hemiglossectomy was performed by the ENT team and subsequent defects were reconstructed using the bilobed LAFF (Fig. 2B). The bilobed LAFF was designed to be about $8 \times 4.5 \mathrm{~cm}$. Upper lobe is for the mouth floor defect and lower lobe for the tongue tip. Posterior radial collateral artery and its vena comitans are used as the pedicle (Fig. 2C). The flap pedicle was anastomosed to the superior thyroidal artery and external jugular vein. Neurorrhaphy was not performed. Postoperatively, a thin LAFF was observed on the hemiglossectomy defect (Fig. 2D). The flap survived without complications (Fig. 2E). At the last follow-up, 5 months postopera- tively, the patient reported no difficulty in tasting and swallowing, and reported some difficulty in pronouncing. In this patient, neurorrhaphy was not performed, but the difficulty in tasting was less seem to the fact that the hemiglossectomy defect was smaller than the other cases.

\section{DISCUSSION}

Tongue reconstruction is challenging with the unique structure and functions like articulation, deglutition, and taste [13]. Ideal reconstruction starts from replacing the deficient tissue with similar size, volume, and texture $[4,8,14]$. For decades, tongue reconstruction was done with primary closure, skin grafts, and pedicled flaps like infrahyoid myofascial flap, pectoralis major myocutaneous flap, or the trapezius islanded pedicle flap. With the inherent inconvenience and limitations, tongue reconstruction was very challenging merely recreating the deficient tissue itself.

Nowadays, microsurgical advancement has explosively delivered various options like rectus abdominis myocutaneous flap, the latissimus dorsi myocutaneous flap, the RFFF, the ulnar forearm flap, the ALTFF, and the medial sural artery perforator flap. The challenge comes down to perfecting the cosmesis and function, not just replacement of deficient tissue. As microsur- 

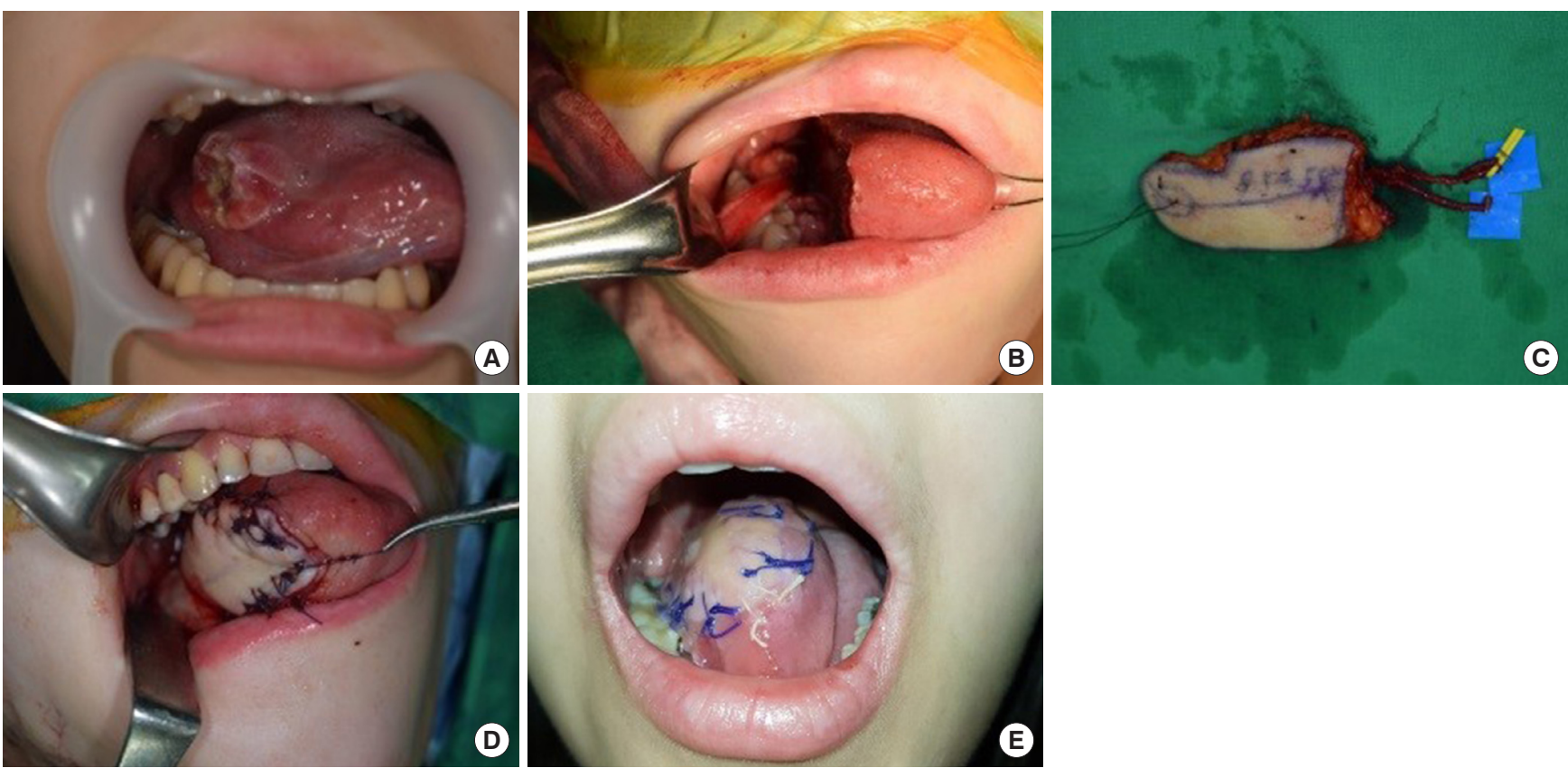

Fig. 2. Case of right hemi-tongue reconstruction using bilobed lateral arm free flap. (A) Preoperative photograph shows biopsy confirmed right tongue cancer. (B) Intraoperative photograph after right hemiglossectomy was performed by the ENT (ear, nose, and throat) department. (C) Intraoperative photograph of harvested bilobed lateral arm free flap. Upper lobe is for the tongue base and lower lobe for the tongue tip. Posterior radial collateral artery and its vena comitans are used as the pedicle. (D) Immediate postoperative photograph of the reconstructed tongue. (E) One-month postoperative photograph of the reconstructed tongue.

gical techniques have developed to a certain level, the nature and innate qualities of flap donor site itself has become the crucial factor that influences the outcome.

Hence, most reports focus on flap selection in regard of tongue defect classification [6,7]. Cheng's classification of hemi, subtotal, two-third, three-quarter, and total tongue defect is widely accepted [8]. Motility is emphasized in small defects, while bulk is emphasized in large defects.

Reconstruction of larger defects target for restoring sufficient volume, as even the simplest role of swallowing, oral competence, and macro-aesthetic appearance cannot be accomplished without bulk. Anterolateral thigh (ALT) flaps are popularly used as with its reliability, long pedicle, acceptable donor site mobility, and versatility as perforator flaps or musculocutaneous flaps [6].

Successful tongue reconstruction of smaller defects depends on thinness, pliability of flap and maintaining tongue mobility. Applying the thinnest possible flap is important because tongue motility comprises of complex arrangements, which is impossible to recreate with musculocutaneous flaps [8]. RFFFs have been the first choice for small to hemiglossectomy defect reconstruction with its thin, pliable, hairless, reliable pedicle, and easy harvest [15]. Although only applicable in patients of thin skin, ALT perforator free flaps are also popularly used as alternative options.
Two unsolved drawbacks of RFFFs come from inevitable skin graft for closure and the fact that a major artery (radial artery) has to be sacrificed. Especially, forearm disfigurement has more negative psychosocial impacts in Asian cultures [16]. Furthermore, no long-term studies are reported regarding sacrifice of a major artery in the upper limb [17].

LAFF, first described in 1982 by Song et al. [9], uses the inessential posterior radial collateral artery as the pedicle. It is known for the thin, pliable, and hairless flap with primarily closable donor site lesser than $7 \mathrm{~cm}$ in width [16]. Average pedicle length is $8.90 \mathrm{~cm}$ [18]. LAFF can be the optimal option for hemi-tongue reconstruction over RFFF for the following reasons. Hair shaft diameter is slightly thinner [19]. Flap thickness is thinner than that of ALT flaps ( $6.32 \mathrm{~mm}$ vs. $9.8 \mathrm{~mm}$ ). Though LAFF is reported to be thicker than RFFF ( $6.32 \mathrm{~mm}$ vs. $2.8 \mathrm{~mm}$ ), our experience reveals that extremely thin septocutaneous flaps can be harvested through a more lateral epicondyle oriented approach than conventional one $[18,20,21]$. It was especially useful for tongue tip reconstruction where thin pliable flap characteristics play bigger roles in shaping the delicate dimension of the tip, providing more functional tongue mobility.

The unique characteristic of LAFF is the striking thickness difference with $2 \mathrm{~mm}$ in the thinnest portion (usually near the lateral epicondyle), $20 \mathrm{~mm}$ in the thickest portion (usually near the deltoid region) [22]. Bilobed flap design utilizing this ex- 

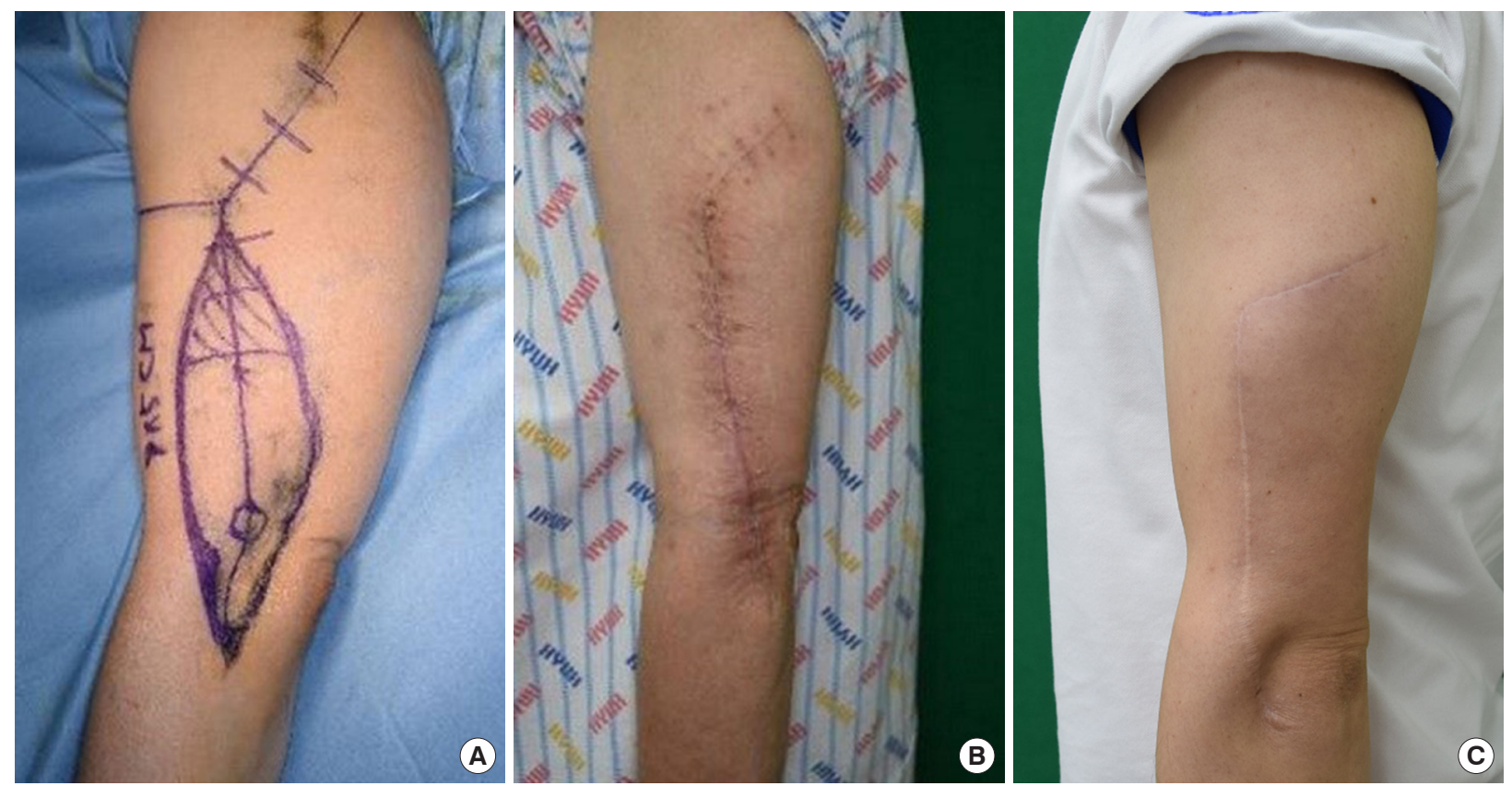

Fig. 3. Primarily closed donor site scar of bilobed lateral arm free flap used for tongue reconstruction, in different periods: (A) preoperative design, (B) 1 month, (C) 7 years.

traordinary feature allows custom-fit reconstruction, thin tongue tip and bulky tongue base and/or mouth floor.

Hemi-tongue reconstruction never requires flap width larger than $7 \mathrm{~cm}$, which allows primary closure of LAFF donor site. Donor site sacrifices the inessential posterior radial collateral artery, and the scar is hidden under short sleeve shirts (Fig. 3). This is valuable for Asian cultures compared to RFFF, as mentioned above. Moreover, patient satisfaction of donor site (all primarily closed) was identical to that of ALT, which is well known for the minimal donor site morbidity [10].

In the past, limitations of LAFF was reported to be sensory loss of donor site, variable anatomy, short and small caliber pedicle. We found these factors to be less highlighted for hemi-tongue reconstruction. Sensory loss of donor site can be prevented through posterior antebrachial cutaneous nerve preservation [21]. Even with the sensory change that can occur, patient satisfaction is not lower than that of ALT [10]. This is probably because the lateral arm area is not exposed frequently. Inconstant anatomy is a misbelief, as Chang et al. [16] has reported. This is in accordance with our finding. The short and small caliber pedicle does not limit cases of tongue reconstruction $[1,9,23]$.

Further issues can expand to sensory aspects of the reconstructed tongue, which is usually often neglected. Currently, there is no consensus on the need for reinnervation of the reconstructed tongue [4]. It is however rational to attempt for sensate flaps, especially for the frequently exposed tongue tip. Sensate RFFF and ALT flaps result in early sensory recovery
$[24,25]$. Nonsensate RFFF shows sensory recovery while ALT, fibular, and jejunal flaps don't [4]. This could be due to the difference of flap thickness, like the fast sensory recovery of fasciocutaneous to musculocutaneous flaps [12].

Despite the ongoing controversy regarding sensate flaps, Biglioli et al. [26] and Katou et al. [27] did report higher satisfaction rates of sensate compared to nonsensate RFFF. Tincani et al. [28] reported better tongue symmetry using sensate compared to nonsensate flaps, which could facilitate swallowing. LAFF could have advantages in sensate flap harvest with the $100 \%$ sensory posterior antebrachial nerve.

Our study could be limited with the small case number and lack of objective measurements. However, LAFF focused exclusively on hemi-tongue reconstruction with tongue tip included without trouble and complaints is meaningful. Future studies with more attempts of LAFF could further elucidate the safety and optimal qualities.

The underrated LAFF is probably optimal for hemi-tongue reconstructions. Well established advantages like thinness, pliability, hairlessness, potentiality of sensate flap, and availability of primary donor site closure are well applicable, without major vessel sacrifice. Misbeliefs or seemingly disadvantageous characteristics like inconstant anatomy, donor site morbidity, short and small caliber pedicle does not cause any burden in hemitongue reconstruction. We suggest bilobed LAFF design as the 1st choice flap for hemiglossectomy defect reconstruction. 


\section{NOTES}

\section{Conflict of interest}

No potential conflict of interest relevant to this article was reported.

\section{Ethical approval}

The study was approved by the Institutional Review Board of Hanyang University Hospital (IRB No. HYUH 2018-04-009) and performed in accordance with the principles of the Declaration of Helsinki. Written informed consent was obtained.

\section{Patient consent}

The patients provided written informed consent for the publication and the use of their images.

\section{ORCID}

$\begin{array}{ll}\text { Jeongseok Oh } & \text { https://orcid.org/0000-0003-2001-084X } \\ \text { Tae Hyeon Lee } & \text { https://orcid.org/0000-0002-2806-3885 } \\ \text { Jang Hyun Lee } & \text { https://orcid.org/0000-0002-0552-4554 } \\ \text { Kyung Tae } & \text { https://orcid.org/0000-0002-0382-2072 } \\ \text { Seong Oh Park } & \text { https://orcid.org/0000-0001-8990-0635 } \\ \text { Hee Chang Ahn } & \text { https://orcid.org/0000-0002-6810-5752 }\end{array}$

\section{REFERENCES}

1. Ganly I, Goldstein D, Carlson DL, Patel SG, O’Sullivan B, Lee N, et al. Long-term regional control and survival in patients with "low-risk," early stage oral tongue cancer managed by partial glossectomy and neck dissection without postoperative radiation: the importance of tumor thickness. Cancer 2013;119: 1168-76.

2. Liu J, Wu H, Zhu Z, Wu X, Tan H, Wang K. Free anterolateral thigh myocutaneous flap for reconstruction of soft tissue defects following en block resection of tongue cancer. Zhongguo Xiu Fu Chong Jian Wai Ke Za Zhi 2010;24:82-6.

3. Tesseroli MA, Calabrese L, Carvalho AL, Kowalski LP, Chiesa F. Discontinuous vs. in-continuity neck dissection in carcinoma of the oral cavity: experience of two oncologic hospitals. Acta Otorhinolaryngol Ital 2006;26:350-5.

4. Baas M, Duraku LS, Corten EM, Mureau MA. A systematic review on the sensory reinnervation of free flaps for tongue reconstruction: does improved sensibility imply functional benefits? J Plast Reconstr Aesthet Surg 2015;68:1025-35.

5. Bokhari WA, Wang SJ. Tongue reconstruction: recent advances. Curr Opin Otolaryngol Head Neck Surg 2007;15:202-7.

6. Chepeha DB, Teknos TN, Shargorodsky J, Sacco AG, Lyden T, Prince ME, et al. Rectangle tongue template for reconstruction of the hemiglossectomy defect. Arch Otolaryngol Head Neck Surg 2008;134:993-8.

7. Hsiao HT, Leu YS, Liu CJ, Tung KY, Lin CC. Radial forearm versus anterolateral thigh flap reconstruction after hemiglossectomy: functional assessment of swallowing and speech. J Reconstr Microsurg 2008;24:85-8.

8. Engel H, Huang JJ, Lin CY, Lam W, Kao HK, Gazyakan E, et al. A strategic approach for tongue reconstruction to achieve predictable and improved functional and aesthetic outcomes. Plast Reconstr Surg 2010;126:1967-77.

9. Song R, Song Y, Yu Y, Song Y. The upper arm free flap. Clin Plast Surg 1982;9:27-35.

10. Klinkenberg M, Fischer S, Kremer T, Hernekamp F, Lehnhardt M, Daigeler A. Comparison of anterolateral thigh, lateral arm, and parascapular free flaps with regard to donor-site morbidity and aesthetic and functional outcomes. Plast Reconstr Surg 2013;131:293-302.

11. Reinert $S$. The free revascularized lateral upper arm flap in maxillofacial reconstruction following ablative tumour surgery. J Craniomaxillofac Surg 2000;28:69-73.

12. Close LG, Truelson JM, Milledge RA, Schweitzer C. Sensory recovery in noninnervated flaps used for oral cavity and oropharyngeal reconstruction. Arch Otolaryngol Head Neck Surg 1995;121:967-72.

13. Elfring T, Boliek CA, Winget M, Paulsen C, Seikaly H, Rieger JM. The relationship between lingual and hypoglossal nerve function and quality of life in head and neck cancer. J Oral Rehabil 2014;41:133-40.

14. Chang EI, Yu P, Skoracki RJ, Liu J, Hanasono MM. Comprehensive analysis of functional outcomes and survival after microvascular reconstruction of glossectomy defects. Ann Surg Oncol 2015;22:3061-9.

15. Soutar DS, McGregor IA. The radial forearm flap in intraoral reconstruction: the experience of 60 consecutive cases. Plast Reconstr Surg 1986;78:1-8.

16. Chang EI, Ibrahim A, Papazian N, Jurgus A, Nguyen AT, Sua$\mathrm{mi} \mathrm{H}$, et al. Perforator mapping and optimizing design of the lateral arm flap: anatomy revisited and clinical experience. Plast Reconstr Surg 2016;138:300e-306e.

17. Huang $\mathrm{CH}$, Chen HC, Huang YL, Mardini S, Feng GM. Comparison of the radial forearm flap and the thinned anterolateral thigh cutaneous flap for reconstruction of tongue defects: an evaluation of donor-site morbidity. Plast Reconstr Surg 2004; 114:1704-10.

18. Busnardo FF, Coltro PS, Olivan MV, Faes JC, Lavor E, Ferreira $\mathrm{MC}$, et al. Anatomical comparison among the anterolateral thigh, the parascapular, and the lateral arm flaps. Microsurgery 2015;35:387-92. 
19. Otberg N, Richter H, Schaefer H, Blume-Peytavi U, Sterry W, Lademann J. Variations of hair follicle size and distribution in different body sites. J Invest Dermatol 2004;122:14-9.

20. Hsu KC, Tsai WH, Ting PS, Hsueh JH, Chen LW, Lin YS. Comparison between anterolateral thigh, radial forearm, and peroneal artery flap donor site thickness in Asian patients: a sonographic study. Microsurgery 2017;37:655-60.

21. Ki SH. Lateral arm free flap with preservation of the posterior antebrachial cutaneous nerve. Ann Plast Surg 2016;76:517-20.

22. Ahn HC. Revision of lateral arm free flap; can it be a substitute for radial forearm free flap? Arch Reconstr Microsurg 1997;6: 80-6.

23. Yang XD, Zhao SF, Wang YX, Li W, Zhang Q, Hong XW, et al. Use of extended lateral upper arm free flap for tongue reconstruction after radical glossectomy for tongue cancer. Aesthetic Plast Surg 2015;39:562-9.

24. Yu P. Reinnervated anterolateral thigh flap for tongue recon- struction. Head Neck 2004;26:1038-44.

25. Loewen IJ, Boliek CA, Harris J, Seikaly H, Rieger JM. Oral sensation and function: a comparison of patients with innervated radial forearm free flap reconstruction to healthy matched controls. Head Neck 2010;32:85-95.

26. Biglioli F, Liviero F, Frigerio A, Rezzonico A, Brusati R. Function of the sensate free forearm flap after partial glossectomy. J Craniomaxillofac Surg 2006;34:332-9.

27. Katou F, Shirai N, Kamakura S, Ohki H, Motegi K, Andoh N, et al. Intraoral reconstruction with innervated forearm flap: a comparison of sensibility and reinnervation in innervated versus noninnervated forearm flap. Oral Surg Oral Med Oral Pathol Oral Radiol Endod 1995;80:638-44.

28. Tincani AJ, Del Negro A, Araujo PP, Akashi HK, Neves Fda S, Martins AS. Head and neck reconstruction using infrahyoid myocutaneous flaps. Sao Paulo Med J 2006;124:271-4. 